The following manuscript is a pre-print version of the original article

Sachana, M., Sidiropoulou, E., Flaskos, J., Harris, W., Woldehiwet'Z., and Hargreaves, A.J. (2014) Diazoxon disrupts the expression and distribution of $\beta$ III-tubulin and MAP 1B in differentiating N2a cells. Basic and Clinical Pharmacology and Toxicology 114, 490-496.

DOI: $10.1111 / \mathrm{bcpt} .12192$ 


\section{Diazoxon disrupts the expression and distribution of $\beta$ III-tubulin and MAP $1 B$ in differentiating $\mathbf{N} 2 \mathrm{a}$ cells}

Magdalini Sachana ${ }^{a}$, Erasmia Sidiropoulou ${ }^{b}$, John Flaskos ${ }^{\text {a, d }}$, Wayne Harris ${ }^{c}$, Alex J. Robinson $^{\mathrm{c}}$, Zerai Woldehiwet ${ }^{\mathrm{b}}$, Alan J. Hargreaves ${ }^{\mathrm{c}, \mathrm{d}}$

${ }^{a}$ Laboratory of Biochemistry and Toxicology, School of Veterinary Medicine, Aristotle University of Thessaloniki, 54124 Thessaloniki, Greece

${ }^{\mathrm{b}}$ Department of Infection Biology, Institute of Infection \& Global Health, University of Liverpool, Leahurst Campus, Leahurst, Neston, Wirral CH64 7TE, UK

c Interdisciplinary Biomedical Research Centre, School of Science and Technology, Nottingham Trent University, Nottingham, NG11 8NS, UK

${ }^{\mathrm{d}}$ Corresponding authors:

Tel: +44 $1158488061 \quad$ Email address: alan.hargreaves@ntu.ac.uk (AJ Hargreaves)

Tel: +30 $2310999948 \quad$ Email address: $\quad$ flaskos@vet.auth.gr $\quad(\mathrm{J}$ Flaskos) 
Abstract: This study aimed at assessing the effects of diazoxon (DZO), a major metabolite of the insecticide diazinon (DZ), on key cytoskeletal proteins in differentiating N2a neuroblastoma cells. Initial experiments established that sub-lethal concentrations of 1, 5 and $10 \mu \mathrm{M}$ DZO produced profound inhibition of neurite outgrowth. Densitometric scanning of probed immunoblots of N2a cell lysates demonstrated that DZO had no effect on total $\beta$-tubulin levels. However, probing with a monoclonal antibody that recognised specifically the $\beta$ III-tubulin isotype revealed that $10 \mu \mathrm{M}$ DZO induced a significant reduction in the levels of this particular form. Levels of polyglutamylated tubulin were not altered. Exposure to $10 \mu \mathrm{M}$ DZO also decreased the expression of microtubule associated protein 1B (MAP 1B). However, DZO had no effect on the expression of MAP tau. DZO also failed to affect the levels neurofilament light (NFL) and neurofilament medium (NFM) chain levels. Indirect immunofluorescence demonstrated that the staining of neurites in treated cells was weaker than in the controls for BIII-tubulin. In conclusion, DZO disrupts the microtubule (MT) network affecting the expression and distribution of two specific MT proteins known to be important in neuritogenesis. DZO may contribute to the developmental neurotoxicity seen following exposure to DZ.

Keywords: Diazoxon; N2a neuroblastoma; Neurite outgrowth inhibition; $\beta$ III-tubulin; MAP 1B; Organophosphorothionate insecticide

Abbreviations: AChE, acetylcholinesterase; CP, chlorpyrifos; DMEM, Dulbecco's modified Eagle's medium; DMSO, dimethyl sulphoxide; DZ, diazinon; DZO, diazoxon; FITC, fluorescein isothiocyanate; HRP, horseradish peroxidase; MAP 1B, microtubule associated protein 1B; MT, microtubule; NF, neurofilament; NFL, neurofilament light chain; NFM, neurofilament medium chain; OP, organophosphorus ester; PAGE, polyacrylamide gel electrophoresis; PBS, phosphate buffered saline; SDS, sodium dodecyl sulphate; TRITC, tetramethyl rhodamine isothiocyanate. 


\section{Introduction}

Organophosphorus esters (OPs) comprise the largest group of insecticides globally employed; however, due to their toxicity, they can cause damaging effects on mammals, birds and the environment in general. OPs can induce in adults a number of distinct neurotoxic effects [1], among which the most frequently encountered is acute neurotoxicity due to the inhibition of acetylcholinesterase (AChE). In recent years, however, an increasing number of in vivo studies indicate that OP insecticides, and particularly the extensively used organophosphorothionates chlorpyrifos (CP) and diazinon (DZ), can also cause developmental neurotoxicity [2, 3]. This toxicity is related to the ability of these OPs to interfere with a number of neurodevelopmental events including the process of neuronal differentiation and axon formation $[4,5]$.

Phosphorothionates are known to be metabolically converted in vivo to their desulphurated, oxon analogues by specific mixed function oxidases (cytochrome p450s), mainly in the liver [6]. In vitro studies using a variety of cell culture systems demonstrate that the oxon metabolites of both $\mathrm{CP}$ and DZ have the capacity to directly disrupt the process of neurite outgrowth, a widely employed marker of neuronal differentiation in vitro. Thus, the oxon metabolite of $\mathrm{CP}$, chlorpyrifos oxon (CPO) inhibits the outgrowth of axonal processes in primary cultures of embryonic rat superior cervical [7] and dorsal root [8] ganglia neurons. CPO can also impair the extension of neurites in the rat PC12 pheochromocytoma [9] and mouse N2a neuroblastoma [10] cell lines. The ability to disrupt the process of neurite development in the N2a cell line is also exhibited by the oxon metabolite of DZ, diazoxon (diazoxon, DZO).

The neuronal cytoskeleton, and in particular microtubules (MTs) and neurofilaments (NFs), is one of the most important endogenous factors that control normal neurodevelopment including the process of axonal/neurite outgrowth [11]. This study sought to assess in differentiating mouse N2a neuroblastoma cells the effects of DZO on the expression and distribution of a number of MT and NF proteins which are neurodevelopmentally important and may be involved in neurite outgrowth and stability. 
DZO was employed at three different concentrations, 1, 5 and $10 \mu \mathrm{M}$, which were found to cause extensive impairment of neurite outgrowth. These DZO concentrations were previously shown to be non-cytotoxic in our laboratory using the same differentiating cell line [12]. 


\section{Materials and methods}

Materials. The mouse neuroblastoma cell line was obtained from ICN (Thane, UK). Cell culture plastic materials were supplied by Scientific Laboratory Supplies (Nottingham, UK). Cell culture reagents, as well as mouse monoclonal antibodies to total $\alpha$-tubulin (B512), total $\beta$-tubulin (TUB 2.1), $\beta$ III-tubulin (SDL.3D10) and polyglutamylated tubulin (clone B3) were purchased from Sigma-Aldrich Co. Ltd (Poole, UK). Mouse monoclonal antibody to glyceraldehyde-3-phosphate dehydrogenase (GAPDH; sc-32233), rabbit polyclonal antibody against MAP 1B (H-130; sc-25729) and goat polyclonal antibodies against tau (C17; sc-1995), NFL (N-14; sc-12966) and NFM (G-20; sc-16143) were obtained from Santa Cruz Biotechnology (CA, USA). Anti-phosphorylated NFH mouse monoclonal antibody SMI34 was supplied by Sternberger Monoclonals Inc. (Berkeley, USA). Horseradish peroxidase (HRP)-conjugated anti-mouse, anti-rabbit and anti-goat secondary antibodies were bought from DakoCytomation (Ely, UK). Fluorescein isothiocyanate (FITC)-conjugated goat anti rabbit immunoglobulin and tetramethyl rhodamine isothiocyanate (TRITC)-conjugated rabbit anti-mouse immunoglobulin were also purchased from DakoCytomation. Diazoxon (purity 97.6\%) was supplied by Chem Service Inc. (West Chester, PA, USA). All other chemicals and reagents were the best grade available from Sigma-Aldrich Co. Ltd (Poole, UK).

Growth and maintenance of N2a cells. N2a cells were maintained as monolayers in the logarithmic phase of growth at $37^{\circ} \mathrm{C}$ in a humidified atmosphere of $5 \% \mathrm{CO}_{2}$ and $95 \%$ air employing growth medium comprising Dulbecco's modified Eagle's medium (DMEM) supplemented with $10 \% \mathrm{v} / \mathrm{v}$ foetal calf serum, $1 \mathrm{mM}$ glutamine, penicillin $\mathrm{G}(100 \mathrm{U} / \mathrm{ml})$ and streptomycin $(100 \mu \mathrm{g} / \mathrm{ml})$, as described previously $[14,15]$ and sub-cultured at 60$80 \%$ confluence. 
Induction of $N 2$ a cell differentiation and DZO treatment. N2a cells, between passage 10 and 20, were detached by pipette and harvested by centrifugation. Cell pellets were resuspended in $1 \mathrm{ml}$ growth medium and cell number determined in a haemocytometer chamber. For determination of neurite outgrowth, N2a cells, detached at 60-80\% confluence, were plated out in 24-well culture dishes at a density of $5 \times 10^{4}$ cells $/ \mathrm{ml}$, with each well receiving $0.5 \mathrm{ml}$ of cell suspension. The cells were allowed to recover for $24 \mathrm{~h}$ in a $\mathrm{CO}_{2}$ incubator and then, to induce differentiation, the growth medium was carefully withdrawn and replaced with serum-free medium containing $0.3 \mathrm{mM}$ dibutyryl cAMP and with or without DZO. Cells were returned to the incubator and allowed to differentiate for $24 \mathrm{~h}$. DZO was freshly prepared as 200 -fold concentrated stock solutions in dimethyl sulphoxide (DMSO) and added to the serum-free medium. The final DMSO concentration in the medium of DZO-treated and untreated control cells was $0.5 \% \mathrm{v} / \mathrm{v}$. In the present study, DZO was employed at final concentrations of 1,5 and $10 \mu \mathrm{M}$. These concentrations have been previously shown to non-cytotoxic towards the differentiating $\mathrm{N} 2 \mathrm{a}$ cells, as determined by two different cell viability assays [12].

Assessment of neurite outgrowth. Following differentiation for $24 \mathrm{~h}, \mathrm{~N} 2 \mathrm{a}$ cell monolayers were fixed with 90\% methanol in Tris-buffered saline (TBS; $10 \mathrm{mM}$ Tris, $140 \mathrm{mM} \mathrm{NaCl}, \mathrm{pH}$ 7.4) and stained with Coomassie Brilliant Blue R-250. They were then washed with TBS and viewed with the aid of an inverted light microscope [14]. Alternatively, they were stained with anti-tubulin or anti-NFH antibodies as described below. From each well, five randomly selected fields giving a total cell count of 200-300 cells/well were examined. In each well the total number of cells and the total number of axon-like processes were counted and the mean number of axon-like processes per 100 cells was calculated. An axon-like process was defined as an extension with a length greater than two cell body diameters [16].

Gel electrophoresis and Western blotting. For Western blotting analysis, N2a cells were seeded in T25 culture flasks in a volume of $10 \mathrm{ml}$ growth medium at a density of $5 \times 10^{4} / \mathrm{ml}$. They were then induced to differentiate in the absence or presence of 1,5 or 10 
$\mu \mathrm{M}$ DZO, as described above. At the end of the differentiation period and prior to cell lysis samples were checked microscopically for proper neurite development (untreated control cells) and neurite outgrowth inhibition (DZO-treated cells). Intact cell monolayers were then solubilised by boiling in $1 \mathrm{ml}$ electrophoresis sample buffer containing 62.5 $\mathrm{mM}$ Tris, 2\% w/v sodium dodecyl sulphate (SDS), 10\% w/v $\beta$-mercaptoethanol and $0.002 \% \mathrm{w} / \mathrm{v}$ bromophenol blue. The resultant cell lysates were subsequently subjected to gel electrophoresis in the presence of sodium dodecyl sulphate (SDS/PAGE) employing either a $7.5 \% \mathrm{w} / \mathrm{v}$ or a $10 \% \mathrm{w} / \mathrm{v}$ polyacrylamide resolving gel, as appropriate, overlaid with a 4\% w/v stacking gel [17]. Separated proteins were then transferred electrophoretically onto nitrocellulose membrane filters [18]. The resultant Western blots were checked for equal protein loading and efficient transfer by staining with $0.05 \% \mathrm{w} / \mathrm{v}$ copper pthalocyanine [19] and were then blocked with $3 \% \mathrm{w} / \mathrm{v}$ non-fat milk powder (Marvel) in phosphate-buffered saline (Marvel/PBS) containing 0.05\% w/v Tween-20 for at least $1 \mathrm{~h}$ at room temperature. The blots were then probed overnight at $4{ }^{\circ} \mathrm{C}$ with appropriate dilutions of primary antibodies in Marvel/PBS. The antibodies included the mouse anti-total $\beta$-tubulin (dilution 1:1000), mouse anti- $\beta$-tubulin III (1:1000), antipolyglutamylated tubulin, rabbit anti-MAP1B (1:200), goat polyclonal anti-tau (dilution 1:500), goat anti-NFL (dilution 1:200), goat anti-NFM (1:100) and anti-GAPDH (1:1000). Following six 10-min washes with PBS containing 0.05\% w/v Tween 20 (PBS/Tween), the blots were probed with appropriate dilutions of HRP-conjugated anti-mouse, antirabbit or anti-goat secondary antibodies for $3 \mathrm{~h}$ at room temperature. After six further washes with PBS/Tween, antibody reactivity was visualised with enhanced chemiluminescence reagent (ECL). For quantification of antibody reactivity, densitometric scanning of images from probed Western blots was performed by using the Quantiscan image analysis system (Version 3; Biosoft), as described previously [19]. The band densities for total $\beta$-tubulin, $\beta$ III-tubulin, polyglutamylated tubulin, MAP $1 \mathrm{~B}$, tau, NFL and NFM were normalised to band densities for GAPDH.

Indirect immunofluorescence staining of N2a cell monolayers. For this, $200 \mu 1$ of cell suspension at $10^{4}$ cells $/ \mathrm{ml}$ were seeded into cell culture chamber slides. Following recovery, cells were induced to differentiate for $24 \mathrm{~h}$ in the absence and presence of 10 
$\mu \mathrm{M}$ DZO, after which cell monolayers were fixed for $10 \mathrm{~min}$ at $-20^{\circ} \mathrm{C}$ in $90 \%$ methanol in PBS, blocked and incubated with either anti- $\alpha$-tubulin, anti- $\beta$ III-tubulin, anti-MAP 1B or anti-phosphorylated NFH (all diluted at 1:200 in $3 \% \mathrm{w} / \mathrm{v}$ BSA in PBS) and either FITC- or TRITC-conjugated secondary antibodies (diluted 1:100 in 3\% w/v BSA in PBS) as described previously [20], except that the anti-fade mountant contained the nuclear staining agent DAPI (Vector Laboratories Inc.)

Statistical analysis. Quantitative data were expressed as mean \pm SEM for at least 3 independent experiments. Statistical analysis for multiple comparisons was performed using one way ANOVA with a post-hoc Tukey test, using $95 \%$ confidence limits. Otherwise the Mann Whitney U-test was employed.

\section{Results}

Preliminary experiments showed that exposure for $24 \mathrm{~h}$ to DZO concentrations of 1,5 and $10 \mu \mathrm{M}$ used in this study produced approximately $50-75 \%$ inhibition of neurite outgrowth (data not shown), which was similar to earlier data [12]. This was further confirmed by measurement of axon-like processes in cells stained with antibodies to total $\alpha$-tubulin and phosphorylated NFH (Fig 1).

In order to reveal the nature of the biochemical phenomena that underlie these potent effects of DZO on cell morphology, Western blots of extracts of N2a cells exposed to the above oxon concentrations for $24 \mathrm{~h}$ were probed with a number of antibodies that are known to recognise key proteins of the neuronal cytoskeleton, since the latter is important in cell morphology and the process of neurite outgrowth. As can be seen in the probed blots in fig. 2, the cross-reactivity of $\mathrm{N} 2$ a cell lysates with a monoclonal antibody that recognises total $\beta$-tubulin was apparently unaffected by treatment with $\mathrm{DZO}$, at all concentrations employed. In contrast, probing of these blots with a monoclonal antibody that recognises specifically isotype III of $\beta$-tubulin revealed that extracts of N2a cells that had been exposed to higher concentrations of DZO exhibited decreased cross-reactivity compared to untreated controls. On the other hand, cross-reactivity with an antibody 


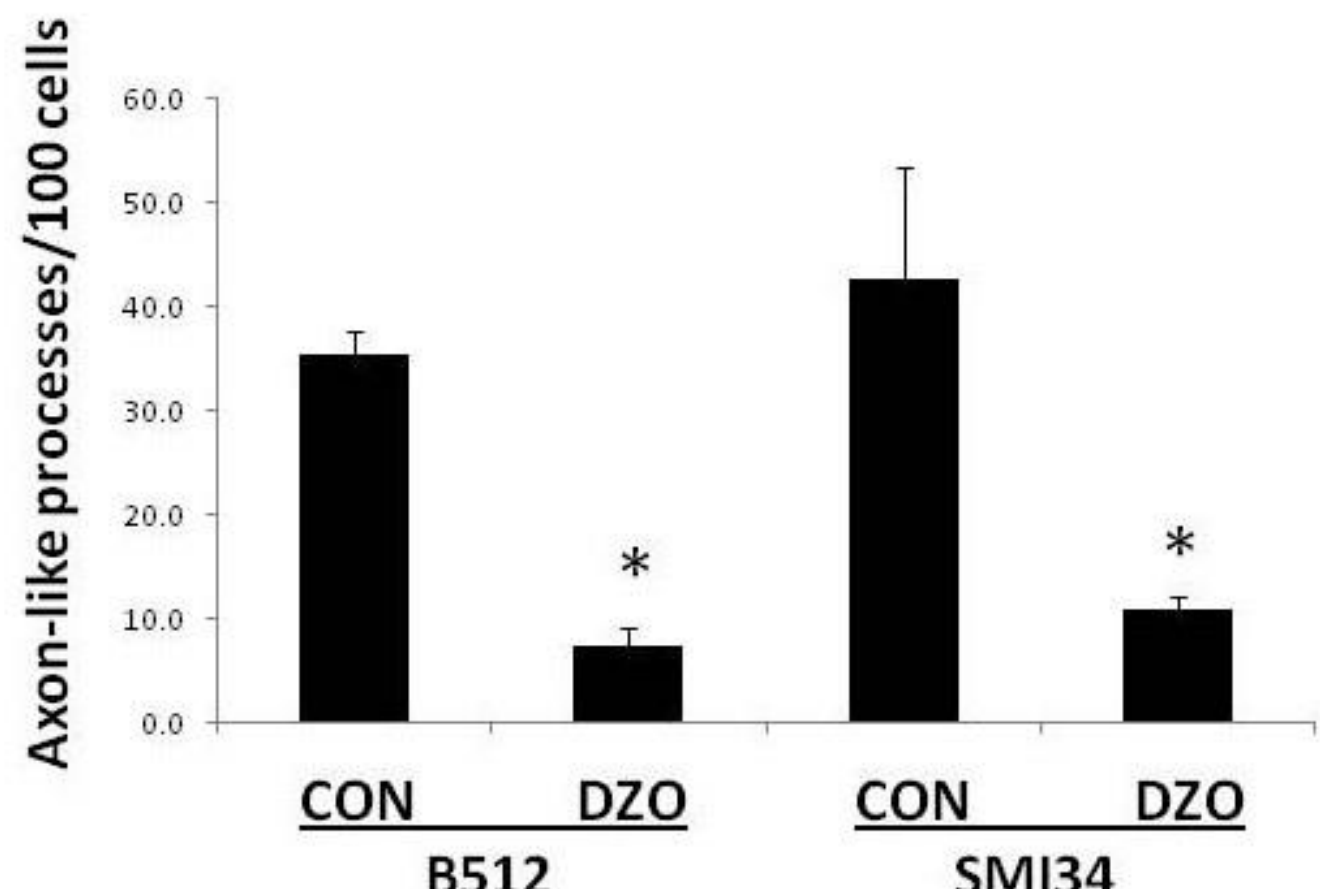

Fig. 1. Effects of diazoxon on neurite outgrowth. N2a cells were induced to differentiate in the absence (control) and presence of $10 \mu \mathrm{M} \mathrm{DZO}$, stained with antibodies to total $\alpha$-tubulin (B512) and phosphorylated NFH (SMI34) and assessed for neurite outgrowth as described in Materials and methods. Shown are mean numbers of axon like neurites per 100 cells \pm S.E.M. Asterisks indicate significant differences compared to the corresponding control $(\mathrm{p}<0.05)$.

recognising polyglutamylated tubulin seemed to be unchanged following DZO treatment. By contrast, cross-reactivity of blots with an antibody recognising MAP 1B appeared to be reduced in DZO-treated cells compared to controls. On the other hand, exposure of $\mathrm{N} 2 \mathrm{a}$ cells to all DZO concentrations for $24 \mathrm{~h}$ had no apparent effect on the levels of cross-reactivity with an antibody recognising tau protein. Finally, probing of blots with antibodies recognising the NF proteins NFL and NFM demonstrated no apparent differences in cross-reactivity between DZO treated samples and controls (data not shown).

Quantification of the observed alterations in antibody reactivity was subsequently carried out employing Quantiscan image analysis software. Densitometric analysis confirmed the 

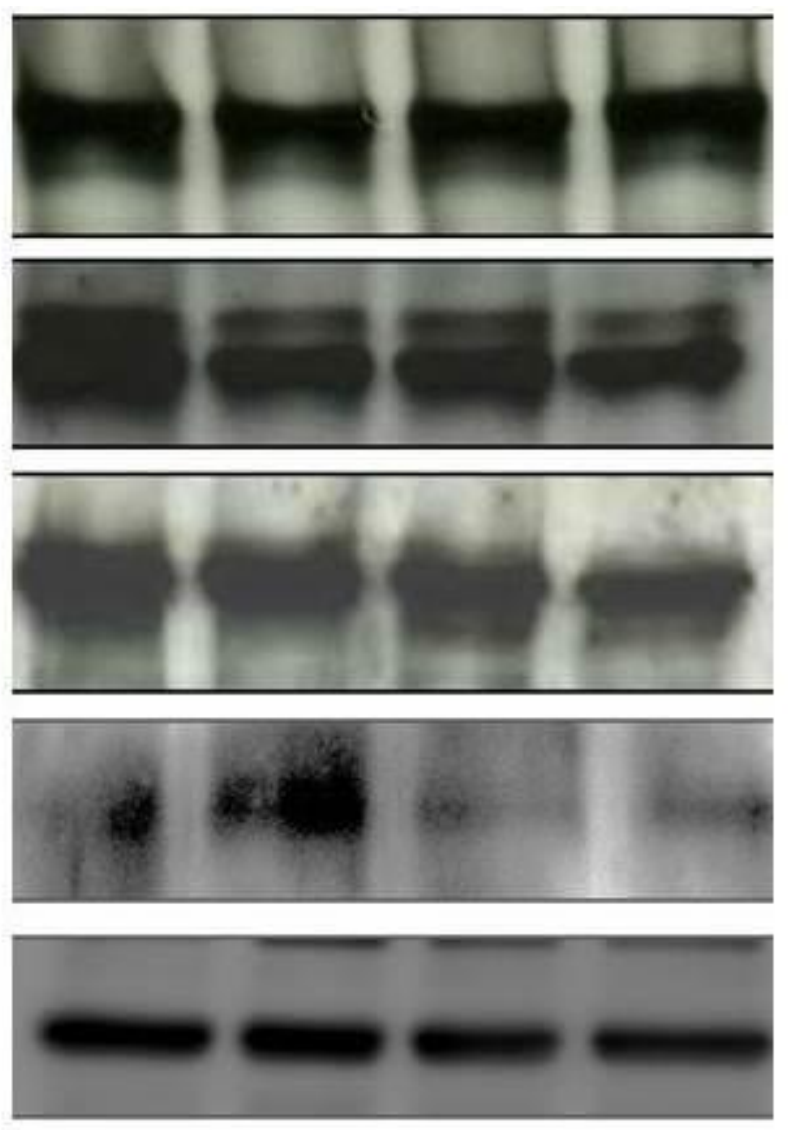

\title{
Total $\beta$-tubulin
}

\author{
Blll-tubulin
}

MAP 1B

\section{Tau}

\section{Polyglutamylated tubulin}

Fig. 2. Western blotting analysis of proteins in control and DZO-treated cell extracts. N2a cells were induced to differentiate for $24 \mathrm{~h}$ in the absence or presence of 1,5 and $10 \mu \mathrm{M}$ DZO prior to extraction of the cell monolayers. Proteins were then separated by SDS-PAGE, subjected to Western blotting and the resultant blots probed with monoclonal antibodies against total $\beta$-tubulin, $\beta$ III-tubulin and MAP1B, as described in Materials and methods. Shown are representative digital images taken using the FUJIFILM LAS-3000 ECL camera. Blots probed with antibodies against the two neurofilament proteins for which the densitometric analysis demonstrated no changes, are not shown.

lack of statistically significant changes in the levels of total $\beta$-tubulin, polyglutamylated tubulin and tau (table 1) as well as in those of NFL and NFM (not shown) following treatment of $\mathrm{N} 2 \mathrm{a}$ cells with 1-10 $\mu \mathrm{M}$ DZO. In contrast, the quantitative analysis revealed that exposure of differentiating N2a cells to $10 \mu \mathrm{M}$ DZO for $24 \mathrm{~h}$ caused a statistically significant 38.6 percent reduction in the levels of the $\beta$ III-tubulin isotype, whereas a reduction of 23.6 percent induced by $5 \mu \mathrm{M}$ DZO was not statistically significant. Densitometric analysis also demonstrated that treatment of $\mathrm{N} 2 \mathrm{a}$ cells for $24 \mathrm{~h}$ with $10 \mu \mathrm{M}$ 


\begin{tabular}{lccc} 
Antigen & & $\begin{array}{c}\text { Densitometric peak area } \\
(\% \text { Control } \pm \text { S.E.M. })\end{array}$ & \\
\cline { 2 - 4 } & $1 \mu \mathrm{M} \mathrm{DZO}$ & $5 \mu \mathrm{M} \mathrm{DZO}$ & $10 \mu \mathrm{M} \mathrm{DZO}$ \\
\hline Total $\beta$-tubulin & $98.4 \pm 10.9$ & $114.8 \pm 4.7$ & $99.0 \pm 5.2$ \\
$\beta$ IIII-tubulin & $114.9 \pm 9.1$ & $76.4 \pm 9.7$ & $61.4 \pm 13.1^{*}$ \\
$\begin{array}{l}\text { Polyglutamylated } \\
\text { tubulin }\end{array}$ & $107.0 \pm 22.3$ & $90.2 \pm 23.1$ & $90.6 \pm 24.7$ \\
MAP 1B & $76.0 \pm 12.6$ & $75.0 \pm 13.4$ & $51.3 \pm 12.5^{*}$ \\
Tau & $149.2 \pm 56.9$ & $129.7 \pm 61.5$ & $133.3 \pm 14.1$
\end{tabular}

Table 1. Densitometric analysis of antibody reactivity on Western blots.

Immunoblots probed with antibodies against total $\beta$-tubulin, $\beta$-III tubulin, polyglutamylated tubulin, MAP1B and tau were incubated with Luminol reagent and the resultant enhanced chemiluminescence was detected as described in Materials and methods. Band densities for all proteins above were normalised to band densities for glyceraldehyde-3-phosphate dehydrogenase. Values are expressed as a percentage of their corresponding control \pm S.E.M. for 3 or 4 independent experiments, each experiment involving a different initial cell population. Asterisks indicate values that were significantly different from their corresponding controls $(* p<0.05)$.

DZO resulted in a statistically significant 48.7 percent decrease in the levels of MAP 1B, whereas 1 and $5 \mu \mathrm{M}$ DZO produced slight but not statistically significant decreases of 24 and 25 percent, respectively.

As shown in fig. 3, indirect immunofluorescence staining with monoclonal antibodies B512 and SMI34 showed that there were less axon-like outgrowths in DZO treated cells than in control cells (see also fig. 1). Furthermore, the intensity of antibody staining in neurites and cell bodies was relatively weak in DZO treated cells compared to the control for anti- $\beta$ III-tubulin but not for anti- $\alpha$-tubulin, with evidence of aggregation in the former. Fewer neurites were also detectable in DZO treated cells stained with anti-MAP1B, 
although the staining intensity was very weak even in control cells (not shown). Staining with the anti-phosphorylated NFH antibody SMI34 also confirmed the appearance of cell body aggregate staining in DZO treated cells, indicative of neurofilament disruption.

\section{Discussion}

The densitometric data obtained in this study indicate that concentrations of DZO which severely impair neurite outgrowth in N2a cells have no effect on the levels of total $\beta$ tubulin. The fact that total $\alpha$-tubulin levels are also unaffected under identical conditions [12] suggests that DZO exposure has no effect on the overall levels of $\alpha$ and $\beta$ subunits. A similar lack of effect was observed on total $\alpha$-tubulin following exposure of differentiating N2a cells to the parent compound DZ [20].

However, $\beta$-tubulin exists as a number of distinct isotypes [21], of which class III $\beta$ tubulin ( $\beta$ III-tubulin) is the only one that is present almost exclusively in neuronal cells [22] and is one of the earliest neuronal cytoskeletal proteins to be expressed during development $[23,24]$. Western blotting analysis of this $\beta$-tubulin isotype in particular revealed that, in contrast to the lack of its effect on total $\beta$-tubulin, DZO, at a neuriteinhibitory dose of $10 \mu \mathrm{M}$, induced a significant reduction in the levels of $\beta$ III-tubulin.

These data suggest that this effect is specific for $\beta$ III-tubulin since the monoclonal antibody employed for the analysis is known to recognise specifically an epitope located on this isoform and not to cross-react with other tubulin isotypes [25]. The observation of decreased $\beta$ III-tubulin levels at a DZO concentration that has been shown in this study to severely impair neurite outgrowth in $\mathrm{N} 2 \mathrm{a}$ cells is consistent with the findings of studies in other laboratories showing that synthesis of the $\beta$ III-tubulin isotype is crucially involved in microtubule formation during neuritogenesis in vivo and in differentiating mouse neuroblastoma cells $[22,26]$. 


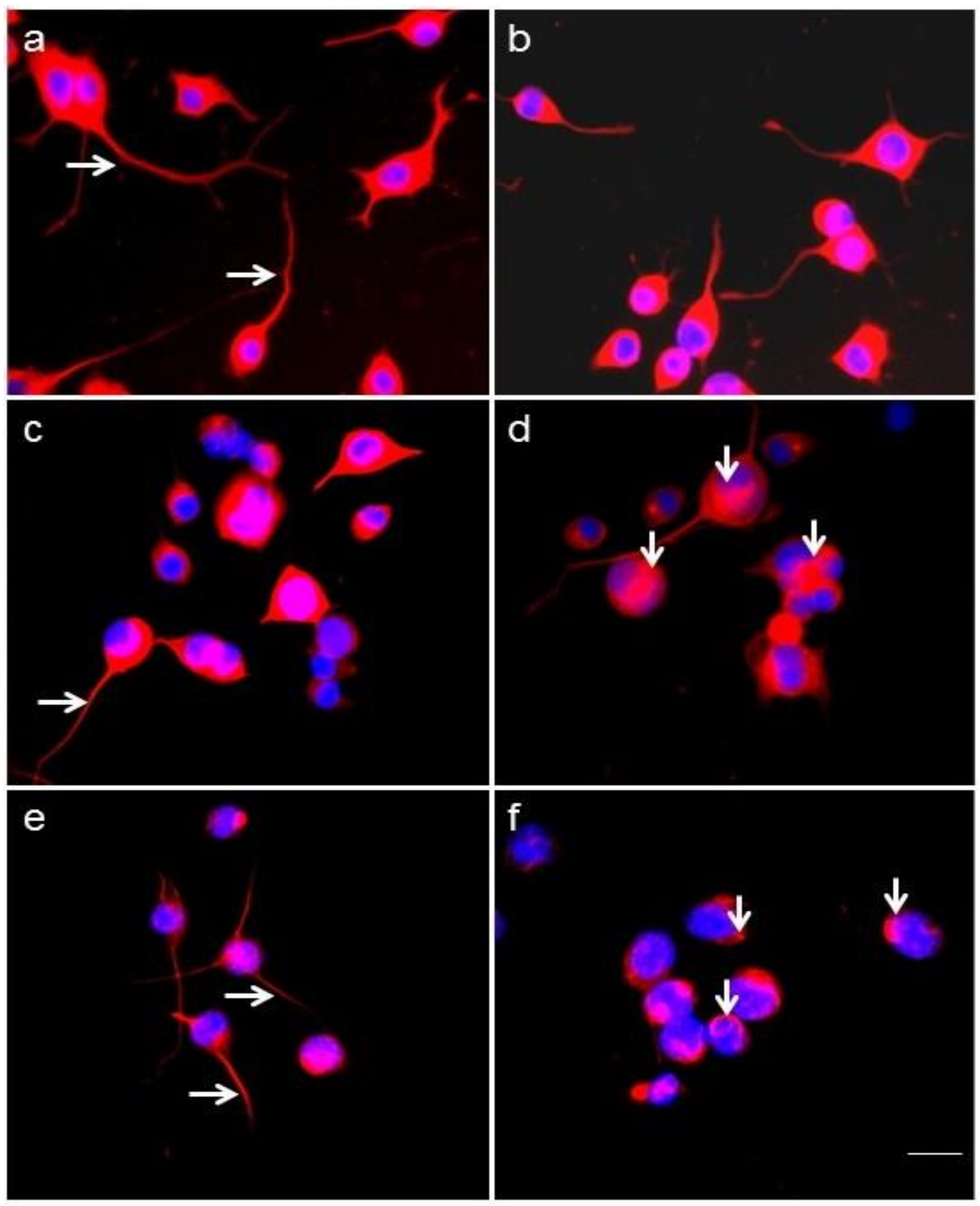

Fig. 3. Indirect immunofluorescence staining of N2a cells induced to differentiate in the absence and presence of DZO. N2a cells were induced to differentiate for $24 \mathrm{~h}$ in the absence (a, c, e) and presence (b, $\mathrm{d}$, f) of $10 \mu \mathrm{M}$ DZO prior to indirect immunofluorescence staining of the cell monolayers as described in Materials and methods. Shown are typical images of staining with anti- $\alpha$-tubulin (a, b), anti- $\beta$ III-tubulin (c, d) and anti-phosphorylated NFH (e, f). Horizontal arrows indicate typical neurites. Downward vertical arrows indicate aggregated staining in cell bodies. Bar represents $20 \mu \mathrm{m}$. 
Reduced levels of $\beta$ III-tubulin could reflect increased degradation by proteases such as calpain, which has been shown to be activated in response to OP treatment in vivo [27]. Alternatively, the reduction in $\beta I I I-$ tubulin levels could be due to decreased synthesis, as phosphorothionates are known to inhibit the synthesis of both DNA and proteins in vivo [28]. The lack of a significant change in the total levels of $\beta$-tubulin in N2a cells treated with $10 \mu \mathrm{M}$ DZO despite a significant reduction in the levels of the $\beta$ III-tubulin isotype can be attributed to the fact that there is a total of five different $\beta$-tubulin isotypes in differentiating mouse neuroblastoma cells [26] and that in these cells the $\beta I I I-t u b u l i n$ is not the main isotype present [29].

Immunoblot analysis also indicated that DZO exposure reduces the levels of the MT stabilising protein MAP 1B. Similarly to $\beta$ III-tubulin, MAP 1B is not uniquely present in the nervous system but is more abundantly expressed in neurons, especially during development [30]. It is the first MAP to be expressed in neurons [31] and plays a key role in neuronal differentiation and the early stages of neuritogenesis [32] when it can act concomitantly or in coordination with $\beta$ III-tubulin [22]. Our present findings showing that doses of DZO which cause neurite inhibition, apart from decreasing the levels of $\beta$ III-tubulin, also reduce at the same time the levels of MAP 1B are consistent with the above data. On the other hand, the fact that the levels of another MAP, tau, were not affected by neurite inhibitory concentrations of DZO, is in line with previous data showing that the retraction of neurites in neuroblastoma cells is not necessarily linked to changes in the amount of tau [33].

Tubulin (poly)glutamylation is a posttranslational modification which is important for binding of tau and other MAPs to tubulin [34]. Glutamylated tubulin accumulates in neuronal cultures and brain during development and may have a role in neurite outgrowth. However, the results suggest that the neurite inhibitory doses of DZO used in this study had no significant effect on the glutamylation state of tubulin. It is worth noting that the level of $\alpha$-tubulin tyrosination, another posttranslational modification of tubulin linked to microtubule stability, was also found to be unaffected in N2a cells by DZO treatment [12]. 
The reduced numbers of long neurites in immunofluorescently stained DZO treated cells confirms our finding that $10 \mu \mathrm{M}$ DZO causes profound inhibition of neurite development, as shown in the morphological analysis presented in fig. 1. However, the reduced intensity of staining of those neurites present with antibodies to $\beta$ III-tubulin but not total $\alpha$-tubulin, suggested a redistribution of $\beta$ III-tubulin. Reduced levels of these proteins in developing neurites may be a key factor in the inhibition of neurite outgrowth by DZO.

In contrast to the considerable changes that DZO induces in the levels of two neurodevelopmentally relevant proteins of the MT system, the effects of this compound on the levels of NFL and NFM, two proteins of the NF network, were not significant. The protein levels of NFH, whose expression in growing neurites occurs later than that of NFL and NFM [11] has also been found to be unaffected by DZO treatment under identical conditions [12]. On the other hand, since the phosphorylation of NFs is an important factor in the regulation of their known ability to modulate axon girth and axon/neurite stabilisation [35], the possibility of neurite-inhibitory doses of DZO exerting a significant effect on NFL and NFM phosphorylation cannot be precluded, particularly in view of our previous data showing that DZO under identical conditions affects the phosphorylation of NFH [12]. Indeed, in the current study, immunofluorescence staining of cells with monoclonal antibody SMI34 (which recognises phosphorylated NFH) confirmed that there was a major redistribution of this network in $\mathrm{N} 2 \mathrm{a}$ cells treated with $10 \mu \mathrm{M}$ DZO. This finding is in good agreement with previous studies using the parent compound DZ [20] and suggests that either reduced levels of NFH or its altered phosphorylation status may be associated with pesticide induced disruption of the NF network.

The significant effects of DZO on the levels of $\beta$ III-tubulin and MAP 1B are caused by a sub-cytotoxic concentration of $10 \mu \mathrm{M}$ and such changes in two neurodevelopmentally important cytoskeletal proteins may be a primary cause of the inhibition of neurite outgrowth induced in N2a cells. On the other hand, DZO concentrations of 1 and $5 \mu \mathrm{M}$ have no significant effect on the above two proteins, although they produce considerable 
neurite outgrowth impairment. Neurite inhibition by these concentrations of DZO in N2a cells has been previously shown to be associated with significant alterations in the levels of the axon growth associated protein GAP-43 and the extent of NFH phosphorylation [12].

Comparison of the effects of DZO with those of its parent phosphorothionate DZ shows that the pattern of cytoskeletal changes related to neurite inhibition is not identical for the two compounds. For example, exposure to both DZ [20] and DZO decreases the levels of MAP 1B in N2a cells. Furthermore, both compounds impair neurite outgrowth without inducing any change in the levels of total $\alpha$-tubulin $[12,20]$. On the other hand, in contrast to DZ, which has been shown to have no effect on the developmentally relevant $\beta$ III-tubulin isotype in N2a cells [36], DZO induces a significant reduction in the levels of this protein, in line with a number of studies indicating a greater range and/or potency of neurodevelopmental effects of the oxon metabolites compared to their parent phosphorothionates [13]. The difference between DZ and DZO is also extended to their effects on proteins of the NF network, as our previous N2a data indicate distinct actions of neurite-inhibitory doses of the two compounds on the levels and phosphorylation of NFH $[12,20]$. Moreover, the differential effects of DZ and DZO on cytoskeletal proteins are not confined to differentiating $\mathrm{N} 2 \mathrm{a}$ cells, but also occur in differentiating cultured cells of glial origin [20,37]. Thus the greater potency of DZO to inhibit neurite outgrowth in differentiating neuronal cells $[12,20]$ may be related to its ability to disrupt additional cytoskeletal protein targets to those affected by DZ.

The above morphological and biochemical effects of DZO noted in vitro are induced by concentrations similar to those likely to occur in vivo in the developing organism. For example, in a prospective cohort study with randomised sampling, one third of foetal meconium samples contained detectable amounts of diazinon with a median value of $12.96 \mu \mathrm{g} / \mathrm{ml}$, which is the equivalent of approximately $40 \mu \mathrm{M}$ [45]. Although studies have not been conducted specifically with DZO, toxicokinetic data obtained for CPO indicate that oxon metabolite levels in the low micromolar range are attainable in the 
developing human [13]. Therefore the above mentioned DZO-specific cytoskeletal changes are relevant to developmental toxicity in humans.

DZO and oxon metabolites in general have a considerably higher aqueous solubility compared to their parent phosphorothionates [38] which could potentially affect their penetration into the foetus. However, data showing significant inhibition of cholinesterase in foetal tissues after in vivo administration of phosphorothionate insecticides [39] indicate that exposure of the foetus to the oxon occurs. In humans, it is thought that the foetus is mainly exposed to oxons formed in maternal tissues. On the other hand, placental metabolism is unlikely to contribute significantly to oxon formation, since CYP2B6, the main CYP responsible for oxon generation in humans [40, 41], is expressed to a limited extent in human placenta [42]. However, although relatively low levels of CYP2B6 are found in the human foetus compared to later developmental stages [43], the levels of the OP degrading enzyme paraoxonase 1 (PON1) are also very low [44] during early development, which could allow some conversion of CPF to CPO within the foetus and/or for the oxon metabolite to accumulate. Additionally, polymorphisms in specific coding and regulatory sequences of the PON1 gene are a major cause of reduced serum PON1 activity and would result in increased risk of susceptibility to the toxicity of the oxon metabolite [44].

In summary, the results of this study indicate that exposure of differentiating cultured cells of neuronal origin to the oxon metabolite of the insecticide DZ causes significant reductions in the levels and distribution specifically of $\beta$ III-tubulin and MAP $1 \mathrm{~B}$, two proteins of the MT network that are known to be important for axonogenesis. These effects are induced by concentrations of DZO which are sub-cytotoxic and toxicologically relevant and they are not related to acetylcholinesterase inhibition [12]. Certain effects produced by DZO are not seen with its parent phosphorothionate DZ. Taken together with our other findings in differentiating C6 cells [37], the present data imply differential targeting by DZO and DZ of key cytoskeletal proteins during neuronal and glial cell differentiation and indicate that the oxon metabolite may contribute 
significantly to the developmental neurotoxicity produced following exposure of the developing organism to the insecticide.

\section{Conflict of interest statement}

The authors declare that there are no conflicts of interest.

\section{Acknowledgements}

The work was supported, in part, by a grant from Pfizer Ltd.

\section{References}

1 Gill KD, Flora G, Pachauri V, Flora SJS. Neurotoxicity of organophosphates and carbamates. In: Satoh T, Gupta RC (eds.), Anticholinesterase Pesticides: Metabolism, Neurotoxicity, and Epidemiology. Wiley, Hoboken, New Jersey, 2010; 237-65.

2 Eaton DL, Daroff RB, Autrup H, Bridges J, Buffler P, Costa LG, et al. Review of the toxicology of chlorpyrifos with an emphasis on human exposure and neurodevelopment. Crit Rev Toxicol 2008; 100: 1-125.

3 Gupta RC, Malik JK, Milatovic D. Organophosphate and carbamate pesticides. In: Gupta RC (ed.), Reproductive and Developmental Toxicology. Elsevier Academic Press, Burlington, MA, 2011; 471-86.

4 Slotkin TA. Developmental neurotoxicity of organophosphates: a case study of chlorpyrifos. In: Gupta RC (ed.), Toxicology of Organophosphate and Carbamate Compounds. Elsevier Academic Press, San Diego, 2006; 293-314.

5 Flaskos J, Sachana M. Developmental neurotoxicity of anticholinesterase pesticides. In: Satoh T, Gupta RC (eds.), Anticholinesterase Pesticides: Metabolism, Neurotoxicity, and Epidemiology. Wiley, Hoboken, New Jersey, 2010; 203-23.

6 Tang J, Rose RL, Chambers JE. Metabolism of organophosphorus and carbamate pesticides. In: Gupta RC (ed.), Toxicology of Organophosphate and Carbamate Compounds. Elsevier Academic Press, Burlington, MA, 2006; 127-43.

7 Howard AS, Bucelli R, Jett DA, Bruun D, Yang D, Lein PJ. Chlorpyrifos exerts opposing effects on axonal and dendritic growth in primary neuronal cultures. Toxicol Appl Pharmacol 2005; 207: 112-24.

8 Yang D, Howard A, Bruun D, Ajua-Alemanj M, Pickart C, Lein PJ. Chlorpyrifos and chlorpyrifos-oxon inhibit axonal growth by interfering with the morphogenic activity of acetylcholinesterase. Toxicol Appl Pharmacol 2008; 228: 32-41.

9 Das KP, Barone SJ. Neuronal differentiation in PC12 cells is inhibited by chlorpyrifos and its metabolites: is acetylcholinesterase inhibition the site of action? Toxicol Appl Pharmacol 1999; 160: 217-30. 
10 Flaskos J, Nikolaidis E, Harris W, Sachana M, Hargreaves AJ. Effects of sublethal neurite outgrowth inhibitory concentrations of chlorpyrifos oxon on cytoskeletal proteins and acetylcholinesterase in differentiating N2a cells. Toxicol Appl Pharmacol 2011; 256: 330-6.

11 Cambray-Deakin MA. Cytoskeleton of the growing axon. In: Burgoyne, R.D. (ed.), The Neuronal Cytoskeleton. Wiley-Liss Inc., New York, 1991; 233-55.

12 Sidiropoulou E, Sachana M, Flaskos J, Harris W, Hargreaves AJ, Woldehiwet Z. Diazinon ox on affects the differentiation of mouse N2a neuroblastoma cells. Arch Toxicol 2009; 83: 373-80.

13 Flaskos J. The developmental neurotoxicity of organophosphorus insecticides: a direct role for the oxon metabolites. Toxicol Lett 2012; 209: 86-93.

14 Flaskos J, McLean WG, Fowler MJ, Hargreaves AJ. Tricresyl phosphate inhibits the formation of axon-like processes and disrupts neurofilaments in cultured mouse N2a and rat PC12 cells. Neurosci Lett 1998; 242: 101-4.

15 Flaskos J, Fowler MJ, Teurtrie C, Hargreaves AJ. Effects of carbaryl and trichlorfon on axon outgrowth in cultured neurons. Toxicol Lett 1999; 110: 79-84.

16 Keilbaugh SA, Prusoff WH, Simpson MV. The PC12 cell as a model for studies of the mechanism of induction of peripheral neuropathy by anti-HIV-I dideoxynucleoside analogs. Biochem Pharmacol 1991; 42: R5-R8.

17 Laemmli UK. Cleavage of structural proteins during the assembly of the head of bacteriophage $\mathrm{T}_{4}$. Nature 1970; 227: 680-5.

18 Towbin H, Staehelin T, Gordon J.. Electrophoretic transfer of proteins from polyacrylamide gels to nitrocellulose sheets: Procedure and some applications. Proc Natl Acad Sci 1979; 76: 4350-4.

19 Sachana M, Flaskos J, Hargreaves AJ. Effects of chlorpyrifos and chlorpyrifosmethyl on the outgrowth of axon-like processes, tubulin and GAP-43 in N2a cells. Toxicol Mech Methods 2005; 15: 405-10.

20 Flaskos J, Harris W, Sachana M, Munoz D, Tack J, Hargreaves AJ. The effects of diazinon and cypermethrin on the differentiation of neuronal and glial cell lines. Toxicol Appl Pharmacol 2007; 219: 172-80.

21 Luduena RF. Multiple forms of tubulin: different gene products and covalent modifications. Int Rev Cytol 1998; 178: 207-75.

22 Katsetos CD, Legido A, Perentes E, Mork SJ. Class III $\beta$-tubulin isotype: A key cytoskeletal protein at the crossroads of developmental neurobiology and tumor neuropathology. J Child Neurol 2003; 18: 851-66.

23 Lee MK, Tuttle JB, Rebhun LI, Cleveland DW, Frankfurter A. The expression and post-translational modification of a neuron specific $\beta$-tubulin isotype during chick embryogenesis. Cell Motil Cytoskeleton 1990; 17: 118-32.

24 Easter SS, Ross LS, Frankfurter A. Initial tract formation in the mouse brain. J Neurosci 1993; 13: 285-99. 
25 Banerjee A, Roach MC, Trcka P, Luduena RF. Increased microtubule assembly in bovine brain tubulin lacking the type III isotype of $\beta$-tubulin. J Biol Chem 1990; 265: 1794-9.

26 Edde B, Jeantet C, Gros F. One $\beta$-tubulin subunit accumulates during neurite outgrowth in mouse neuroblastoma cells. Biochem Biophys Res Comm 1981; 103: 1035-43.

27 El-Fawal HA, Ehrich MF. Calpain activity in organophosphorous-induced delayed neuropathy (OPIDN): Effects of a phenylalkylamine calcium channel blocker. Ann NY Acad Sci 1993; 679: 325-9.

28 Song X, Violin JD, Seidler FJ, Slotkin TA. Modeling the developmental neurotoxicity of chlorpyrifos in vitro: macromolecule synthesis in PC12 cells. Toxicol Appl Pharmacol 1998; 151: 182-91.

29 Gard DL, Kirschner MW. A polymer-dependent increase in phosphorylation of $\beta$ tubulin accompanies differentiation of a mouse neuroblastoma cell line. J Cell Biol 1985; 100: 764-74.

30 Gordon-Weeks PR, Fischer I. MAP1B expression and microtubule stability in growing and regenerating axons. Micr Res Techn 2000; 48: 63-74.

31 Tucker RP, Binder LI, Matus A.. Neuronal microtubule-associated proteins in the embryonic avian spinal cord. J Comp Neurol 1988; 271: 44-55.

32 Gonzalez-Billault C, Owen R, Gordon-Weeks PR, Avila J. Microtubule-associated protein $1 \mathrm{~B}$ is involved in the initial stages of axonogenesis in peripheral nervous system cultured neurons. Brain Res 2002; 943: 56-67.

33 Sayas CL, Avila J, Wandosell F. Regulation of neuronal cytoskeleton by lysophosphatidic acid: role of GSK-3. Biochim Biophys Acta 2002; 1582: 144-53.

34 Fukushima N, Futura D, Hidaka Y, Moriyama R, Tsujiuchi T. Post-translational modifications of tubulin in the nervous system. J Neurochem 2009; 109: 683-93.

35 Sihag RK, Inagaki M, Yamaguchi T, Shea TB, Pant HC. Role of phosphorylation on the structural dynamics and function of types III and IV intermediate filaments. Exp Cell Res 2007; 313: 2098-109.

36 Harris W, Sachana M, Flaskos J, Hargreaves AJ. Neuroprotection from diazinoninduced toxicity in differentiating murine N2a neuroblastoma cells. Neurotoxicology 2009; 30: 958-64.

37 Sidiropoulou E, Sachana M, Flaskos J, Harris W, Hargreaves AJ, Woldehiwet Z. Diazinon oxon interferes with differentiation of rat C6 glioma cells. Toxicol In Vitro 2009b; 23: 1548-52.

38 Sogorb MA, Vilanova E. Detoxication of anticholinesterase pesticides. In: Satoh, T., Gupta RC (eds.), Anticholinesterase Pesticides: Metabolism, Neurotoxicity, and Epidemiology. Wiley, Hoboken, New Jersey, 2010; 121-32. 
39 Gupta RC. Environmental agents and placental toxicity: Anticholinesterases and other agents. In: Sastry BVR (ed.), Placental Toxicology. CRC Press, Boca Raton, 1995; 257-78.

40 Foxenberg RJ, McGarrigle BP, Knaak JB, Kostyniak PJ, Olson JR. Human hepatic cytochrome P450-specific metabolism of parathion and chlorpyrifos. Drug Metab Dispos 2007; 35: 189-93.

41 Croom EL, Wallace AD, Hodgson E. Human variation in CYP-specific chlorpyrifos metabolism. Toxicology 2010; 276: 184-91.

42 Pelkonen O, Vahakangas K, Gupta RC. Placental toxicity of organophosphate and carbamate pesticides. In: Gupta RC (ed.), Toxicology of Organophosphate and Carbamate Compounds. Elsevier Academic Press, Burlington, 2006; 463-79.

43 Croom EL, Stevens JC, Hines, RN, Wallace AD, Hodgson E. Human hepatic CYP2B6 developmental expression: The impact of age and genotype. Biochem Pharmacol 2009; 78, 184-90.

44 Costa L, Vitalone A, Cole TB, Furlong CE. Modulation of paraoxonase (PON1) activity. Biochem Pharmacol 2005; 69, 541-50.

45 Ostrea EM, Morales V, Ngoumgna E, Prescilla R, Tan E, Hernandez E, Baens Ramirez G, Cifra HL, Manlapaz ML. Prevalence of fetal exposure to environmental toxins as determined by meconium analysis. Neurotoxicology 2002; 329-339. 